\title{
A STUDY ON CROSS LAYER METRICS OF WIRELESS MESH NETWORK
}

\author{
Swarnali Chakraborty ${ }^{1}$, Abhishek Majumder ${ }^{2}$ \\ ${ }^{1}$ Department of Computer Science and Engineering, Tripura University \\ ${ }^{2}$ Department of Computer Science and Engineering, Tripura University
}

\begin{abstract}
Wireless mesh network (wmn) offers a flexible and cost effective network infrastructure for wireless communication. But due to wireless environment and unpredictable user mobility, it suffers from the problem of routing path selection, security, scheduling, resource allocation and congestione etc. For solving these problems, several metrics have been proposed. But using information from only one layer the metric does not perform well. So, cross layer mertics have been proposed to achieve a better performance. This paper presents a study on cross layer metrics in the light of their versatile uses for solving different problems in wmn.
\end{abstract}

Keywords: Wireless Mesh Network, Cross layer metrics, Routing, Scheduling.

-***

\section{INTRODUCTION}

Wireless Mesh Network (WMN) emerges as a new and promising technology for the next generation wireless technologies [1][9]. In a WMN, communication among the nodes situated at different location is done by relaying information over wireless links [14]. The Mesh Networks are self-organized, self-configured and easily adaptable to different traffic requirements and network changes [11]. The three different network elements that construct the mesh network architecture are [14]: network gateways, mesh routers and mobile nodes.

Network Gateway (NG): This element acts as an entrance to another network.

Mesh Routers (MR): MRs performs routing of internet as well as intranet traffic to and from the mobile nodes.

Mobile Nodes (MN): The Mobile Node (MN) is a device whose location and point of attachment to the internet may change frequently.

As WMN deal with the highly unstable wireless medium the design of algorithms that consider link quality to choose the best route are enabling routing metrics and protocols to evolve. Cross layer metrics are the new hype of the wireless networks. It has achieved a good result as in the cross layer approach multiple parameters from different layers can intercourse compactly [12].

In this paper some of the different utilities of cross layer metrics have been discussed. The cross layer metrics are used in many areas such as for the efficient routing, to manage the traffic, to identify the selfish participants, etc. This paper shows some of the aspects of the cross layer metrics.
Rest of the paper is organized as follows. Section 2 describes the overview on cross layer metrics. Section 3 presents some of the metric components. Section 4 discusses some of the use of the cross layer metrics and finally in section the conclusion has been presented.

\section{OVERVIEW}

Several metrics are used in WMN for performing a wide range of functionalities. Generally the layered approach is followed. This section gives an overview on the metrics for WMN.

In Open System Interconnection (OSI) model the design and the implementation of the network is divided into modules. Each layer controls its own decision variable. It hides the complexity of the lower layer and provides well defined services to the upper layer. It is easy for debugging, abstraction design and implementation [8]. But it is not good for energy efficiency and also for performance. There is no mechanism for performance optimization between different layers.

To improve the performance interaction between the layers is very much necessary. For this, cross layer approach has been introduced. The main idea is to maintain the functionalities associated to the original layers but allow coordination, interaction and joint optimization of protocols crossing different layers. Cross-layer design refers to protocol design done by allowing layers to exchange state information in order to obtain performance gains. Two approaches for crosslayering can be defined [8]:

- Weak cross-layering: It enables interaction among entities at different layers of the protocol stack. 
- Strong cross-layering: It enables joint design of the algorithms implemented within any entity at any level of the protocol stack.

Figure 1 shows the difference between the layered approach and the cross layer approach

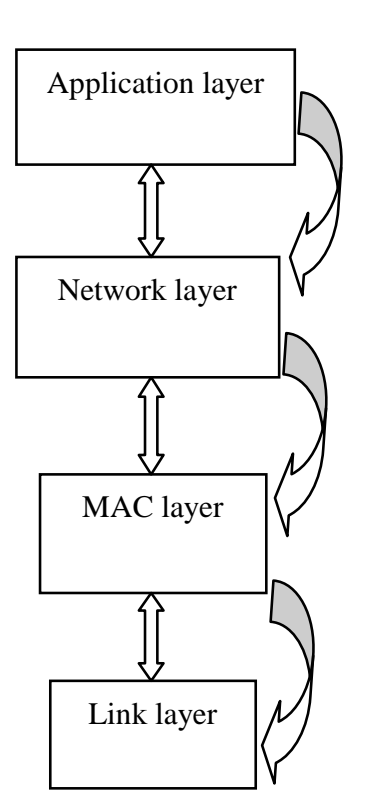

(a)

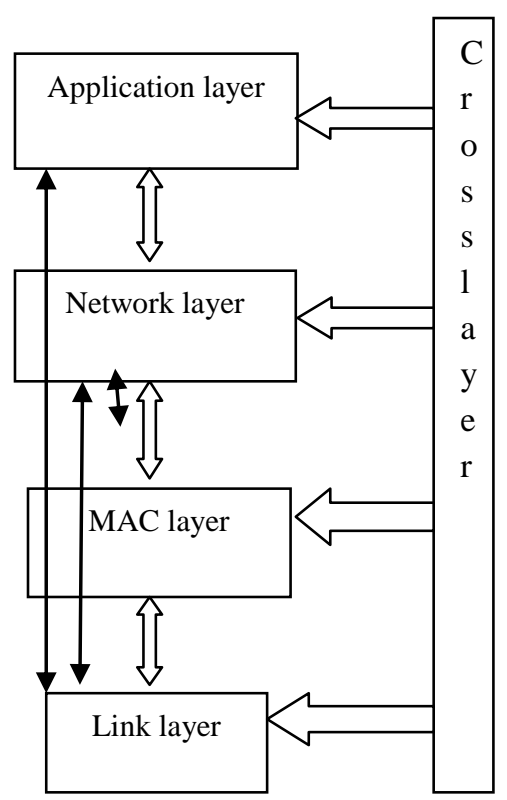

(b)
Fig 1: (a) Layered approach (b) Cross layer approach

\section{METRIC COMPONENTS}

The key components that can be achieved by the metrics are given as [12]:

- Number of Hops: Increase in the number of hops increases the congestion. This component may also be a metric itself.

- Link Capacity: It can be used to measure the throughput of the link. Link capacity measures the total load the link can transfer.

- Link Quality: It gives an idea about the rate at which data can be transferred. It is obvious to choose a high quality link to deliver data at a higher rates and lesser errors.

- Channel Diversity: When the same channel is used within the same path among multiple consecutive hops, co-channel interference may occur. Thus the nodes of the same path should operate on nonoverlapping channel. This is called channel diversity.

\section{CROSS-LAYER METRICS}

Many cross layer metrics have been proposed. The cross layer metrics are useful in many areas for the efficient performance of WMN. Some of its utilities are discussed as below:

\subsection{Cross-Layer Metric for Selfish Participants}

In WMN, there may be some participants that can exhibit selfish behavior by selectively dropping packets sent by other mesh routers to prioritize their own traffic. Stephano Paris et.al. in [4] proposes a cross layer metric, Expected Forwarding Counter (EFW), which combines the link quality measured by the Expected Transmission Counter (ETX) and the forwarding behavior of relaying node. The metric select the path by considering both the quality of the link and the reliability of the nodes. Routing metrics like ETX represent the transmission reliability of the link. ETX calculates the number of transmission, including the retransmissions.

Let $(i, j)$ be a wireless link established between node $i$ and $j, p_{i j}$ and $\mathrm{p}_{\mathrm{ji}}$ denote the packet loss probability of the wireless link ( $\mathrm{i}$, j) in forward and reverse directions, respectively. The probability of a successful transmission on the wireless link (i, j) can therefore be computed as $\mathrm{p}_{\mathrm{s}, \mathrm{ij}}=\left(1-\mathrm{p}_{\mathrm{ij}}\right) \cdot\left(1-\mathrm{p}_{\mathrm{ji}}\right)$.

The expected number of transmission necessary to deliver data can be calculated as [4]-

$$
E T X=\frac{1}{p_{s, i j}}=\frac{1}{\left(1-p_{i j}\right) \cdot\left(1-p_{j i}\right)}
$$

Though ETX selects the most reliable path it does not give the delivery rate of a link accurately as it does not take into account the forwarding behavior of the nodes. The selfish node drop packets of other nodes after receiving the data frame and transmission of acknowledgement successfully. To rectify the dropping behavior of the selfish participants the link quality value calculated by the ETX is combined with the forwarding reliability of a relaying node. It is assumed that $\mathrm{p}_{\mathrm{d}, \mathrm{ij}}$ be the dropping probability of a network node $\mathrm{j}$. Thus the probability of successfully forwarding a packet sent through a node $\mathrm{j}$ is calculated as -

$$
E F W=\frac{1}{p_{f w d, i j}}=\frac{1}{\left(1-p_{i j}\right)\left(1-p_{j i}\right)\left(p_{d i j}\right)}
$$

Thus EFW represents a cross layer metric as it considers both the physical condition of the link and also the selfishness of the nodes.

\subsection{Cross Layer Metric For Routing And Resource}

\section{Allocation}

Wireless mesh network consisted of a number stationary wireless mesh routers. The interference degrades the capacity and performance of WMN. In [2] Taimur Adalgamouni et al. propose joint cross layer routing and resource allocation metric for multi-radio WMN. Frequency bands are assigned to the 
links by the resource allocation algorithm to minimize the interference and maximize the coherence time of the link.

A connection is first established between the source and destination node. The traffic passing through the link is calculated as:

$$
\operatorname{traffic}(l)=\sum_{(c, r) \in s_{1}} \theta_{c} \lambda_{c} x(c, r)
$$

The delay experienced by the link is calculated as:

$$
\operatorname{delay}(l)=\frac{1}{\mu_{1}-\operatorname{traffic}(l)}
$$

A cost function is proposed to minimize the delay and total interference of each link and maximize the coherence time that will minimize the end to end delay and maximize the packet delivery rate.

$$
\begin{aligned}
& \operatorname{delay}(l)+ \\
& \sum_{(c, r) \in s_{1}} x(c, r) \sum_{j \in \operatorname{In} t_{1}} \sum_{f=1 . . L} y(l, f) \rho(l, j, f) y(j, f)+ \\
& \sum_{(c, r) \in s_{1}} x(c, r) \sum_{f=1 \ldots L} \frac{y(l, f)}{\tau(l, f)}
\end{aligned}
$$

Int $_{\mathrm{i}}$ is the set of all links that can interfere with link $1, \mathrm{y}(1, \mathrm{f})$ is a binary control variable that equals to one only if link 1 is assigned frequency band $\mathrm{f}$ and $\tau(1, \mathrm{f})$ is the coherence time of link 1 when assigned band $\mathrm{f}$.

\subsection{Cross Layer Metric for Environmental Congnition}

Huaiyu Wen et al. in [5] have proposed a routing protocol based on Optimised Link State Routing Protocol (OLSR). CMOLSR (Cross Layer Metric based Optimized Link State Routing) is a proactive routing protocol that refines the idea of the cross layer design. The protocol makes use of a routing metric CLM (Cross Layer Metric) which considers four aspects of link quality. They are: bandwidth, load, transmission error rate and wireless interference.

$$
B \omega_{\epsilon}=\frac{B \omega_{\text {available }}}{B \omega_{\text {basic }}}
$$

$\mathrm{B} \omega_{\text {available }}$ denote available bandwidth and $\mathrm{B} \omega_{\text {basic }}$ denote basic data bandwidth.

$$
\operatorname{Load}_{\in}=\frac{Q_{\text {wait }}}{Q_{\text {max }}}
$$

$\mathrm{Q}_{\text {wait }}$ denote the number of data packets waiting to be sent in te sending queue, $\mathrm{Q}_{\max }$ denote the maximum length of waiting queue.

$$
P D R_{\in}=\frac{F_{r e v}}{F_{\text {sent }}}
$$

$\mathrm{F}_{\text {rev }}$ denote number of frames successfully received and $\mathrm{F}_{\text {sent }}$ denote the number of overall frames sent.

$$
L I_{\in}=\frac{|I S(e)|}{I S N}
$$

|IS(e) denote the number of nodes of the set which may have interference with wireless interference. ISN denote the number of nodes contained by the largest IS(e) set.

$$
C L M(e)=\alpha .\left(1-B \omega_{\epsilon}\right)+\beta \cdot \operatorname{Load}_{\epsilon}+\gamma\left(1-P D R_{\epsilon}\right)+\delta \cdot L I_{\epsilon} \alpha, \beta, \gamma, \delta
$$

are the weight factor and $|\alpha|+|\beta|+|\gamma|+|\delta|=1$

The network cross layer design has been followed by the protocol in two ways. In the first design every layer of node share their information with the sharing pool. In the second design the strict hierarchical structure is not followed while separation between layers is maintained and this allows the different algorithms of different layers to exchange their information about the network. The route calculation is done using the Dijkstra algorithm.

\subsection{Congestion Aware Cross Layer Metric for Multipath Network}

Many of the routing protocols of Wireless Mesh Network are mainly dependent on the minimum hop count. But it is not obvious that the path selected by the minimum hop count should be the best one in multipath routing. Because in multipath routing there may be more than one route having the same minimum hop count. To avoid such conditions in [6] Wenming Song et al. proposes two dynamic metric that check the congestion status at nodes. One metric is a quantity called Buffer Occupancy Ratio (BOR) which gives the resource utilization of a node and ability of forwarding packet. Another metric is the Successful Frame Sending Rate (SFSR) which is used to give the contention of an area and the cost for sending packets through that area. The limited resource availability at a node in comparison with the incoming traffic is the main cause for the congestion. BOR is used to calculate congestion status at the node [6]:

$$
\text { BOR }=\text { occupied buffer size/ buffer size }
$$

SFSR is used to reflect both the channel condition and link congestion.

SFSR=Number of received ACKs/Number of sent frames. 
No periodical broadcasting is required to gather the information used to calculate both the metrics as they are available at the nodes or can be collected.

In this routing protocol when a node wants to send data it will check its route table to find whether a route is available or not. If it is available, it will initiate the Route Request (RREQ). The time stamp, SFSR and maximum acceptable delay fields are attached with the RREQ. When a node receives the RREQ it will decide whether it should forward the RREQ. At first, it will check its BOR and will see whether it is becoming congested. Secondly, it calculates the time for which the packet has been survived then it will compare the time with the maximum acceptable delay. If it is less then only the node will forward it otherwise drop it. Thirdly, the node compares its SFSR with the SFSR of the RREQ. If the RREQ SFSR value is larger replace it with its SFSR. After checking whether the packet is valid or not at the destination node a Route Reply (RREP) is initiated. Before sending the RREP the reverse route and SFSR value is recorded into the routing table. When a source node receives the RREP it records the RREP into the routing table and sends the packets through the route.

\subsection{Cross Layer Metric For Scheduling}

Though WMN acts as a backbone for many network applications but it still suffers from scalability, poor end to end throughput, QoS problems. Many scheduling techniques have been proposed but all that mainly focuses only on one characteristic. So a cross layering approach has been followed by Jason B. Ernst in [3]. In this approach information from many layers are gathered and optimized together. In scheduling techniques can be taken from many approaches and optimize together. Scheduling at MAC layer is difficult as there is no proper information for scheduling. So information are gathered from other layers such as queue length, channel quality etc, which are more useful to decide how to allocate time and resources to nodes.

In this approach, scheduling is done against three characteristics. Firstly the biasing is done against the distance from the gateways. The more distance from the gateway the router is, the hop number increases so the probability of successful delivery of packet decreases. Thus to achieve a higher throughput it is good to send fewer packet through the node that is farther away from the gateway. Secondly the biasing is done against queues. The routers with full queues are given priority than the routers with short queue length. The routers with full queue are given priority because it will help the shorter queues to build up and also allow full queue to empty them so that fewer packet are dropped. Thirdly the biasing is done against the poor links. The links with good quality are given priority to achieve a good performance and increase packet delivery rate.
Resources assigned to a node in these three techniques are determined using the equation below.

$$
R=\frac{\alpha}{c^{\beta_{1}}}+\frac{1-\alpha}{c^{\beta_{2}}}
$$

$\mathrm{R}$ is the resource allocated to the node

$\mathrm{c}$ is the measure of a particular characteristic. $\mathrm{c}>0$

$\alpha$ is the weight of each bias technique, $0<\alpha<1$

$\beta_{1}, \beta_{2}$ are biasing constants that determines the strength of each bias.

The last technique is the combined approach where several bias techniques are combined to form a single scheduling approach. In this technique a fraction of the resources is assigned to each of the bias-technique.

The Combined mixed-bias is given as

$$
R=\gamma_{1} R_{1}+\gamma_{2} R_{2}+\gamma_{3} R_{3}
$$

Where $\mathrm{R}$ is the resources allocated to the node $\mathrm{R} 1$ is the resources calculated using above equation. $\gamma 1$ is the weight of technique 1 in the combined biasing $\mathrm{R} 2$ is the resources calculated using above equation $\gamma 2$ is the weight of technique 2 in the combined biasing $\mathrm{R} 3$ is the resources calculated using above equation. $\gamma 3$ is the weight of technique 3 in the combined biasing $\gamma 1, \gamma 2, \gamma 3>0$ and $\gamma 1+\gamma 2+\gamma 3=1$

\subsection{Cross Layer Routing Metric}

A cross layer metric has been proposed by Da Gou et al. in [10] that take into account the physical layer feature, MAC layer feature and then combine them to form a hybrid metric. The metric takes into account the Packet Success Rate (PSR) of the link which denotes the physical link characteristics. The link quality metric is taken from the MAC layer. It is calculated by taking into account the RTS and CTS frame. The quality of a link is dependent on the number of retransmission of the RTS and Data Frame for each successful transmission. The PSR is calculated from the Packet Error Rate (PER):

$$
\mathrm{PSR}=1-\mathrm{PER}
$$

From the physical layer PSR can be calculated and is transmitted to the network layer.

Frame Transmission Efficiency (FTE) is calculated by considering the success rate of transmitting frame based on the average number of retransmission. The number of retransmission of RTS and Data Frame denote the link quality. The quality and the congestion of the link is determined by the number of retransmission of the RTS and Data Frame for each 
successful transmission in MAC layer. The number of retransmission is assumed as:

Failure $(\mathrm{k})=$ ACKFailureCount $(\mathrm{k})+\mathrm{RTSF}$ ailureCount $(\mathrm{k})$.

Ack_Failure_Count denote the number of Data retransmission and RTS_Failure_count denotes the number of RTS retransmission.

Thus FTE is calculated as-

$$
\operatorname{FTE}(k)=\frac{2}{\text { Failure }(k)+2}
$$

To choose the minimum path to transmit packet the minimum hop count is used.

$$
\text { Hopmetric }=\frac{\min \text { hop }}{\text { hopnumber }}
$$

Thus the hybrid metric is expressed as:

$$
\text { Hybridmetric }=\text { Hopmetric } \times \overline{F T E} \times P S R
$$

\subsection{Cross Layer Metric to Recognize Traffic}

Sunghun Lee et al. in [7] have proposed a metric that is useful to select the path with less interference. Wireless Mesh Networks are also called static wireless networks. Communications between the routers are done through the radio nodes. When the two nodes want to transmit packet they should be within the transmission range and no other sending node should be present within that range. If any sending node is present within the same transmission range collision would occur. The nodes at the interference range between source and destination has to wait for the medium to be cleared before accessing it. A metric is proposed to select a path with less congestion, minimum packet loss and less traffic. The MAC architecture is mainly based on the Distributed Coordination Function (DCF), Point Coordination Function (PCF). Here each station maintains a contention window and a back off timer. An initial value for the contention window is set. After each retransmission occured due to collision the value of the contention window is sequentially increased with integer power of 2 and minus 1 . But this technique does not give a good result when the number of transmitting nodes increases. So to overcome such obstacle the traffic recognizing metric transmits data after checking traffic and interference by hearing the RREP. A source node will broadcast a RREQ when it has data to send. When the intermediate node receives the RREQ a route is created to the source. The intermediate node then checks if it is not the destination nor having the path to the destination, it will rebroadcast the RREQ. After the destination node receives the RREQ it generate the RREP and broadcast in a hop-by-hop fashion to the source. Here the pure flooding technique has been followed. When the RREP is received by the intermediate nodes a path is set up from the destination node and the neighboring nodes record the condition of interference by overhearing RREP packets. Here let $\mathrm{TX}_{\mathrm{k}}$ is the number of node that cause interference by generating traffic at sending node; $\mathrm{RX}_{\mathrm{k}}$ the number at receiving node; and $\mathrm{TX}_{\mathrm{k}} \cap$ $\mathrm{RX}_{\mathrm{k}}$ the number of nodes causing interference at the intermediate of link.

$$
\text { path }_{k}=\sum_{\text {linkk }} E T T \times\left|\log \left(T X_{k}\right)+\log \left(R X_{k}\right)+T X_{k} \cap R X_{k}\right| \times \alpha
$$

\subsection{A Cross-Layer Load-Independent Link Cost Metric}

In [13] Marianna Carrera et al. have proposed Cross-layer Unicast Transmission Time (X-UTT), a MAC-aware loadindependent link cost metric for wireless mesh networks. It is designed to capture the wireless link capacity and is independent of the load induced by self-interference and crossinterference in a mesh network. The information acquired from a network-layer unicast probing system and a MAC-layer monitoring system is utilized by the X-UTT metric.

The performance of the Wireless network is mainly dependent on the ability to route packets efficiently over the multi hop wireless mesh infrastructure. The existing metric like ETX, ETT etc are mainly incorporated with the loss of packets, available bandwidth to measure the link quality. The X-UTT is designed to be load-independent and characterize wireless link quality only in terms of the link capacity presented to the network layer by the underlying MAC protocol operation. XUTT is designed on the basis of unicast network-layer probes subjected to MAC-layer (re)transmissions. The sum of the transmission airtime and the backoff time of all its MAC layer (re)transmissions give the packet transmission time of each probe. The transmission air-time information which is a bitrate function, the backoff time prior to each (re)transmission which is a function of the number of previously failed transmissions, are extracted from a MAC layer monitoring system implemented at the wireless card driver.X-UTT is defined as the ratio between the average packet transmission time (PTT) and the unicast probe delivery ratio as $\left(\mathrm{d}_{\mathrm{u} 3}\right)$

$$
X U T T=\frac{P T T}{d_{u 3}}
$$

IN 802.11 MAC layer error control (acks and retransmissions) is same as perceived by the upper layers. X-UTT is designed to capture the quality of 802.11 MAC layer. To hide losses to the higher layers error control mechanism retransmits unacknowledged frames and increases link capacity by increasing the network layer delivery ratio. The transmission time of a network layer packet is the sum of the time spent for all the MAC layer retransmissions. The error control 
mechanism of a network layer decreases the link capacity by increasing the total time to transmit a packet. These opposite effects are captured by the components of X-UTT.

\section{CONCLUSIONS}

The cross layering approach has been followed to discover many metrics for the Wireless Mesh Network. The cross layer approach is adapted to achieve a good performance and to avoid less congested paths. Through cross layering a good idea about the network can be achieved as the information from different layers are optimized compactly. The cross layer metrics are used for routing, to avoid traffic and congestion, to improve the QoS and for many other purposes. In this paper some of the metrics are mentioned. Many more metrics are still to be identified to improve the performance of the wireless mesh network.

\section{REFERENCES}

[1] Luigi Iannon, Ramin Khalili, Kav'e Salamatian, Serge Fdida, "Cross-Layer Routing in Wireless Mesh Networks" $1^{\text {st }}$ International Symposium in Wireless Comuniction Systems, September 2004.

[2] Taimour Aldalgamouni,Ahmed Elhakeem," A Joint Cross Layer Routing and Resource Allocation Algorithm for Multi-Radio WirelessMesh Networks" in the proceedings of IEEE conference on june 2009 pp 349-354.

[3] Jason B. Ernst, Mieso K. Denko," Cross-Layer Mixed Bias Scheduling for Wireless Mesh Networks" in the proc of IEEE conference on May 2010,pp 1-5.

[4] Stefano Paris, Cristina Nita-Rotaru, et al," EFW: A Cross-Layer Metric for Reliable Routing in Wireless Mesh Networks with Selfish Participants" IEEE INFOCOM , PP 576-580,2011.

[5] Huaiyu Wen,Guangchun Luo"Environmental Cognition Based Wireless Mesh Network Cross-layer Routing Protocol" in the proc of Information \& Computational Science 2012,pp 473-487.

[6] Wenming Song,et al,“A Congestion-Aware Multipath Routing with Cross Layer Design for Wireless Mesh Networks" in the proc of $15^{\text {th }}$ Asia Pacific Conference oct.2009,pp 656-660.

[7] Sunghun Lee, Hyukjoon Lee and Hyungkeun Lee, “A Cross Layer Routing Metric To Recognize Traffic Interference in Wireless Mesh Networks" in the proc of of IEEE conference 2010, pp 396-401.

[8] ProfNizar Zorba, Charalambos Skianis and Christos Verikoukis, a book chapter " CROSS LAYER DESIGNS IN WLAN SYSTEMS”

[9] Ian F. Akyildiz, Xudong Wang, Kiyon,"A Survey on Wireless Mesh Networking" IEEE Communications Magazine, vol. 43(9), pp. S23-S30, Sept. 2005.

[10] Da Guo, Jun Li, Mei Song, Junde Song,"A Novel Cross-Layer Routing Algorithm in Wireless Mesh
Network" in the proc. of IEEE International Conference 2007,pp 1-3,vol-07.

[11] B.Nandini, et al,"Cross-Layer Design for wireless mesh networks" in the proc of IEEE Transaction onMobile Computing,2008,pp 1363-1373.

[12] S. Siva Nageswara Rao, Y.K. Sundara Krishna, and K. Nageswara Rao," A Study of Routing Metrics for Wireless Mesh Networks" in the proc of International Journalof Rsearch and Reviews of Wireless Communication 2011.

[13] Marianna Carrera, Henrik Lundgren, Theodoros Salonidis, Christophe Diot "A Cross-Layer LoadIndependent Link Cost Metric for Wireless Mesh Networks" in the proc. 2007 ACM CoNEXT conference.

[14] Jun Yuan,et al,“A Cross-Layer Optimization Framework for Multi hop Multicast in Wireless Mesh Networks" in the proceedings of IEEE on Selected areas in communication, vol.24, no11, November 2006. 